\title{
Article
}

\section{Behavioral Implications in COVID-19 Spread and Vaccinations}

\author{
Karim Chichakly
}

check for

updates

Citation: Chichakly, K. Behavioral Implications in COVID-19 Spread and Vaccinations. Systems 2021, 9, 72. https://doi.org/10.3390/systems9040072

Academic Editor: Alessandro Giuliani

Received: 1 September 2021

Accepted: 12 October 2021

Published: 14 October 2021

Publisher's Note: MDPI stays neutral with regard to jurisdictional claims in published maps and institutional affiliations.

Copyright: (C) 2021 by the author. Licensee MDPI, Basel, Switzerland. This article is an open access article distributed under the terms and conditions of the Creative Commons Attribution (CC BY) license (https:/ / creativecommons.org/licenses/by/ $4.0 /)$.
ISEE Systems, Inc., Lebanon, NH 03766, USA; karim@iseesystems.com

\begin{abstract}
COVID-19 vaccinations have been administered quickly in the USA. However, a surprisingly large number of Americans are unwilling to get vaccinated. Without enough people getting vaccinated, the pandemic will not end. The longer the pandemic persists, the more opportunities exist for more virulent strains to emerge. This model looks at the effects of people's behavior in containing and ending the COVID-19 pandemic in the USA. Human behavior adds several feedback loops to the standard SEIR model. Comparisons are made between cases with and without behavior loops, with reduced adherence to the recommended or mandated masks and social distancing, with and without the vaccine, and the effects of an early mask mandate termination. The results suggest human behavior must be accounted for in epidemiology models and that removing masks before enough vaccine are administered not only puts those vaccinated at risk, but allows the disease to readily spread again.
\end{abstract}

Keywords: COVID-19; vaccinations; SEIR model; human behavior; USA COVID-19 vaccines; pandemic management

\section{Introduction}

In the past year, many models of the spread of COVID-19 have been developed. For example, Struben [1] explored the dynamics of the outbreak and policies to contain it, Ghaffarzadegan and Rahmandad [2] looked at the disparity between reported and actual data in Iran, Sy et al. [3] tied COVID-19 containment policies to economic consequences, and Fair et al. [4] examined the efficacies of a wide variety of testing and contact tracing polices. These studies focused on the spread of the disease and policies to contain it.

However, humans tend to adapt to conditions and evaluate risk based on their perception of current conditions [5]. Few studies have included this impact of human behavior on containing the pandemic. Bogdewic and Ramaswamy [6] used causal-loop analysis to map some of the behavioral links. Rahmandad, et al. [7] included perceived risk and adherence fatigue in their model, but did not include broader issues around vaccinations, e.g., fear of the vaccine and perceived safety based on the number of people vaccinated. The purpose of this research was to explore the impacts of these risk-based perceptions on both the success of the vaccination program and the spread of COVID-19 in the United States of America (USA).

A system dynamics model [8] was built based on the epidemiological susceptibleexposed-infected-recovered (SEIR) model, a variation of the SIR model [9]. The SEIR model is a compartmental model that divides the population into four groups (stocks) based on the infected state of each individual (Figure 1). Susceptible people have never been infected. They become Exposed based on their contact with infected people and the probability of the disease spreading. After the average time it takes someone who has been exposed to show symptoms, they move to the Infected stock. Finally, after the average time to recover, infected people become Recovered. This base model assumes that people cannot be reinfected by the same disease, but it is relatively easy to expand the model to handle this. 


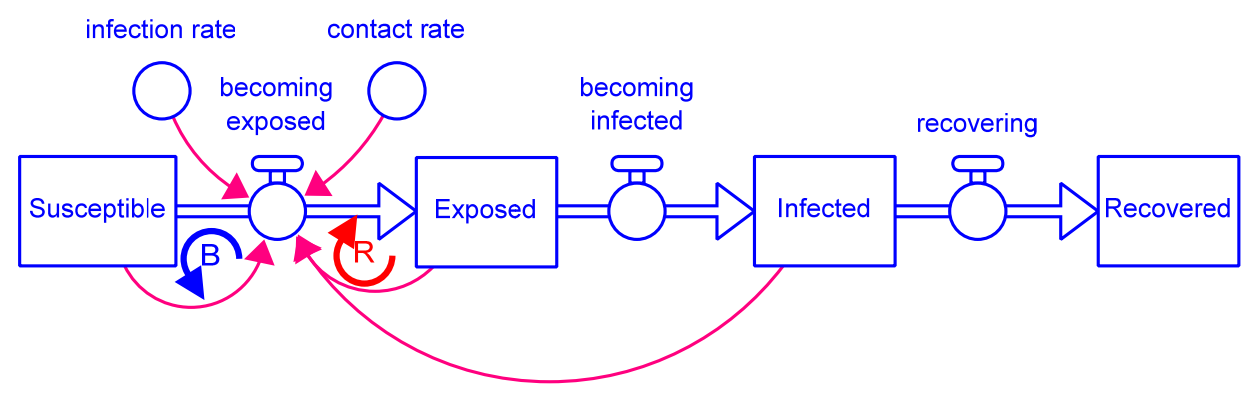

Figure 1. Stocks and direction of flow in the SEIR model.

Two feedback loops control the intrinsic logistic growth of this structure: A reinforcing feedback loop $(R)$ that spreads the disease from those who are Exposed and Infected to those who are Susceptible and a balancing feedback loop $(B)$ that limits the growth when the pool of Susceptible people becomes too small to sustain exponential growth. The parameters that drive the spread of the disease are the average contact rate of people in this population, i.e., how many people each person is in close enough contact with each day to potentially infect them, and the infection rate of the disease itself, i.e., how likely a contacted person will get infected.

\section{Methods}

\subsection{Model Structure}

Since the early days of COVID-19, people who showed symptoms were quarantined. In addition, there were tests available early on to check if asymptomatic individuals (either exposed or infected) were carriers of the virus. Positive tests led to quarantine. Finally, some percentage of the symptomatic people became so ill that they died, rather than recovered. These details led to the addition of stocks for quarantining those who were exposed and those who were infected, as well as a stock of those who died (Figure 2).

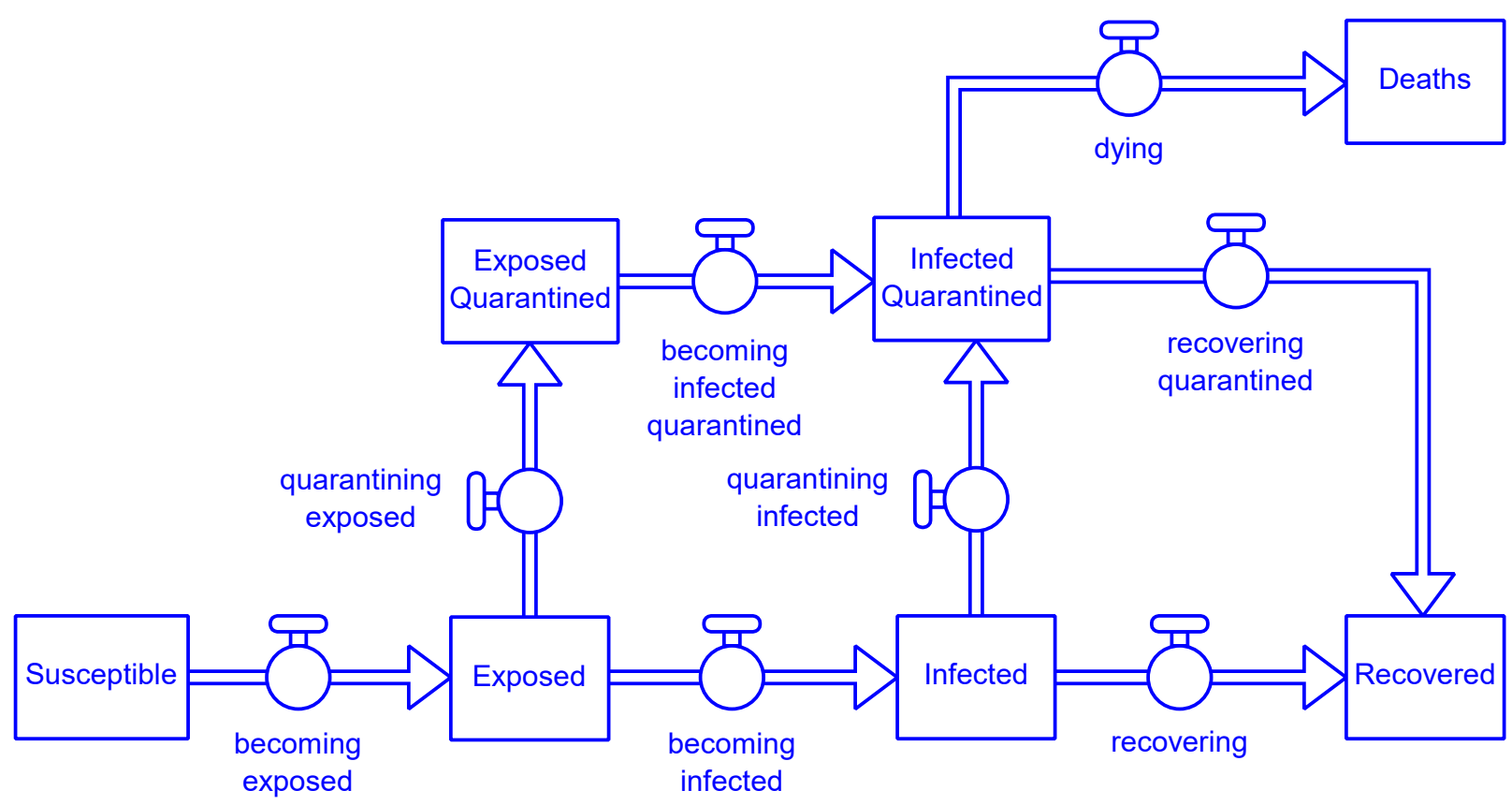

Figure 2. Stocks and flows in the COVID-19 model.

Because the vaccine is not 100\% effective, some people who are vaccinated stay in the Susceptible stock. To ensure they are not vaccinated again, this entire structure is arrayed by Vaccine_Status, which has two elements, not_yet and vaccinated. 
All vaccines modeled require two doses, with a 25-day delay between the doses. This is managed with the structure shown in Figure 3. For simplicity, only those who are susceptible or recovered are vaccinated. Available doses are prorated between them based on their relative sizes (as in a random trial). With the first dose, they enter the Vaccinated Once conveyor (stock with a fixed delay), where they wait the required minimum time to the second dose. Once they have entered this stock, the model does not allow for anyone to either become infected or to infect others. For this reason, there is also no concept of an additional two-week delay before they are "fully vaccinated." While this may seem oversimplified, remember that some vaccinated people remain in the Susceptible stock. To simplify accounting and due to their relatively small numbers, these susceptible vaccinated people do not consume vaccine resources for their second dose.

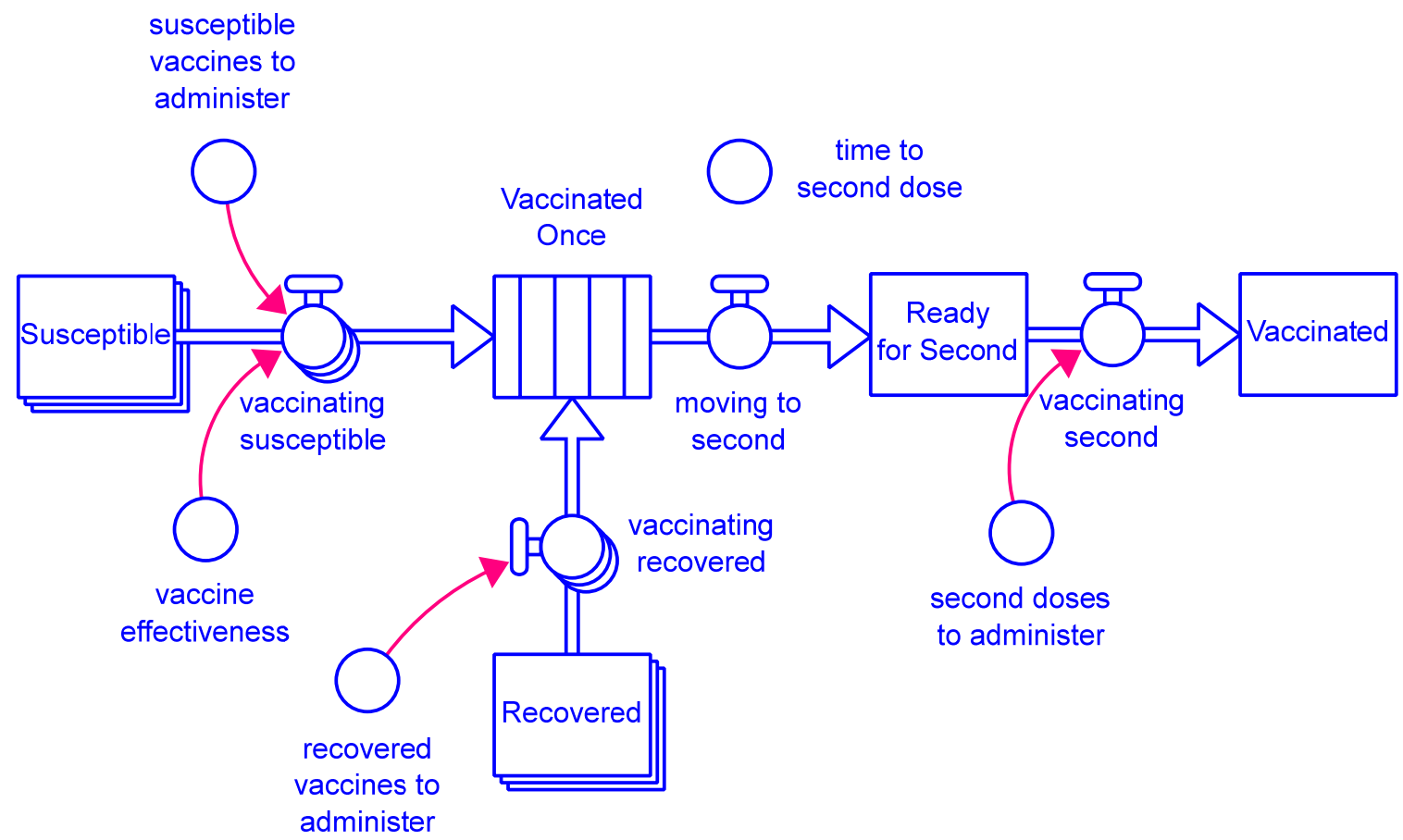

Figure 3. Multiple vaccination management.

Once the minimum time has passed, people move to the Ready for Second stock to wait for a second dose to become available before moving on to the Vaccinated stock. People awaiting a second vaccine dose have priority over those awaiting their first dose.

\subsection{Model Feedback}

Figure 4 shows the five major feedback loops of the behavioral aspects in this model. As new cases decrease, perception of risk decreases after a short delay (indicated by the two parallel lines through the connection). As the perception of risk decreases, people increase their contact with others, and the number of new cases increases (loop B1). Additionally, a decrease in perception of risk leads to people not following suggested or mandated prophylactic measures, such as wearing masks, social distancing, and frequent hand washing, which increases the infection rate, again causing new cases to increase (loop B2).

An increase in new cases gives the virus more opportunities to mutate, leading to more new strains that are more contagious (raising infection rate), leading to even more new cases (loop R1). Within the model, new strains increase as a linear function of new cases. 


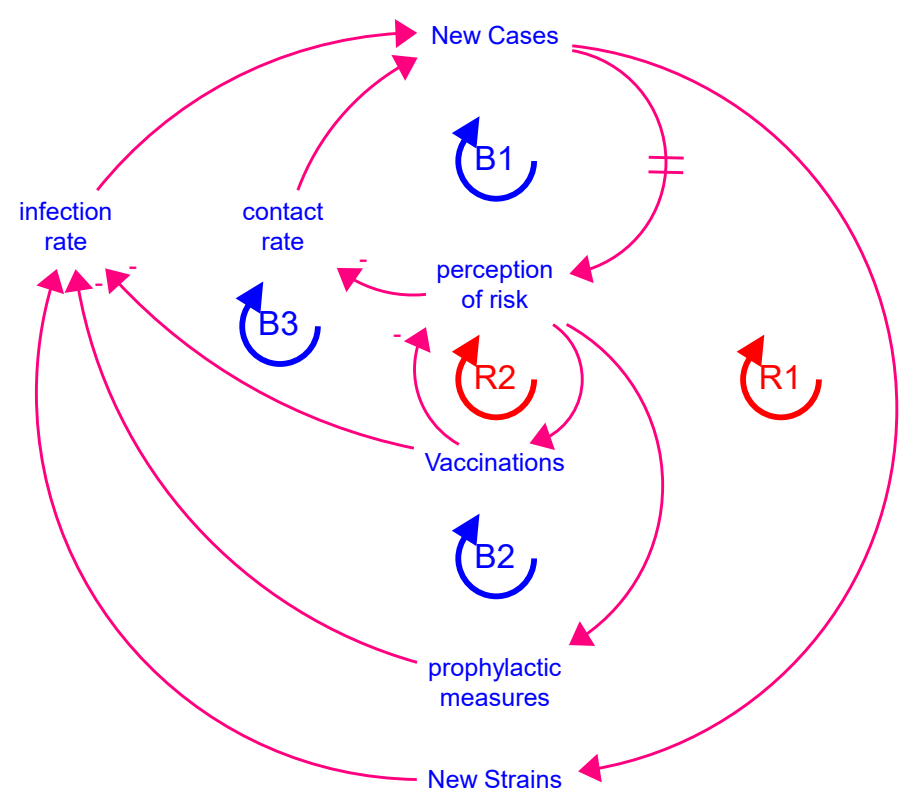

Figure 4. Behavioral feedback in the model. Unmarked polarities are all positive.

As new cases increase and the perception of risk with it, more people get vaccinated, which decreases the contact rate between infected and susceptible people (vaccinated people are neither). This, in turn, lowers the perception of risk, increases the contact rate, and can lead to more new cases (loop R2). Simultaneously, vaccinations fulfill their function: The more people vaccinated, the lower the aggregate infection rate, thus lowering the number of new cases (loop B2).

The delay in the second-order (i.e., two-stock) balancing loop $B 1$ leads to oscillation [8], thus capturing the separate waves of the pandemic. Without such a loop, the model would not oscillate.

There are two additional behavioral effects in the model. First, there is a drifting goals archetype structure [10] around the perception of risk (Figure 5). The drifting goals archetype captures human adaptions of what is perceived as normal in the face of changing circumstances. This is sometimes referred to as "the frog in the frying pan" effect, that is, as the frying pan gets hotter, the frog's perception of what temperature is normal increases with the frying pan's temperature until it gets hot enough to kill the frog without the frog ever recognizing the danger. In the COVID-19 situation, people become inured to the larger numbers of new cases and deaths as the pandemic unfolds, so the level of new cases at which they perceive risk continually increases with the expansion of the pandemic.

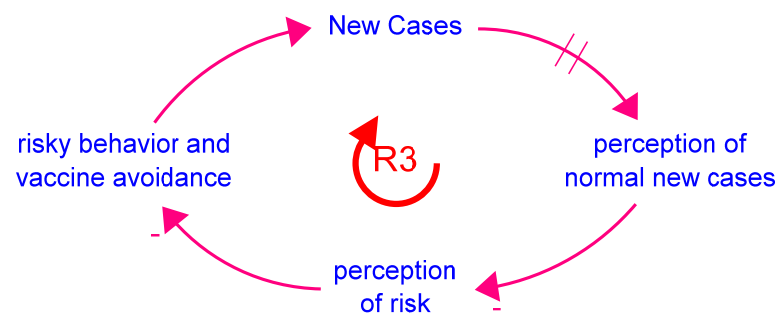

Figure 5. Drifting goals' impact on risk perception. Unmarked polarities are all positive.

Second, there has been a large degree of vaccine hesitancy in the USA. One prominent cause during the early period of vaccinations was the distrust that the vaccine was tested adequately and therefore may be unsafe or not effective (leading to a false sense of security against infection and possible death). Other causes include association of vaccines with liberal democrats, minimization of the severity of the disease (e.g., "It is no worse than a cold"), privacy concerns, mistrust by Black Americans due to the Tuskagee experiments, 
etc. The fear of the vaccine is represented in the model as shown in Figure 6. As more people are safely vaccinated, the fear decreases until vaccine hesitancy-at least for the reason of vaccine distrust-is gone.

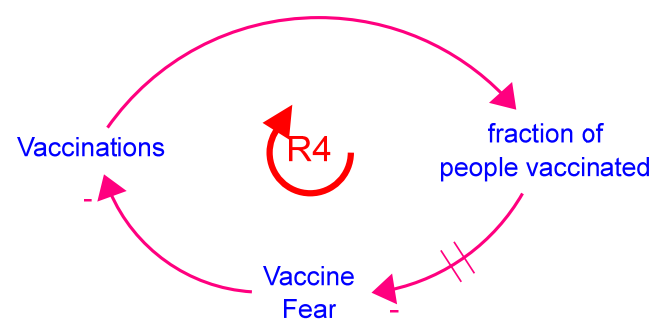

Figure 6. Vaccine hesitancy from fear of the vaccines not being safe decreases with the fraction of people vaccinated.

A seasonal effect has also been included. During warmer weather and holidays, people mingle with each other more, increasing the contact rate.

Figure 7 shows the behavioral loops (all feedback loops but the mutation loop, R1) in the context of the full model structure. For readability, the connections that go to both vaccinating susceptible and vaccinating recovered are only shown going to vaccinating susceptible.

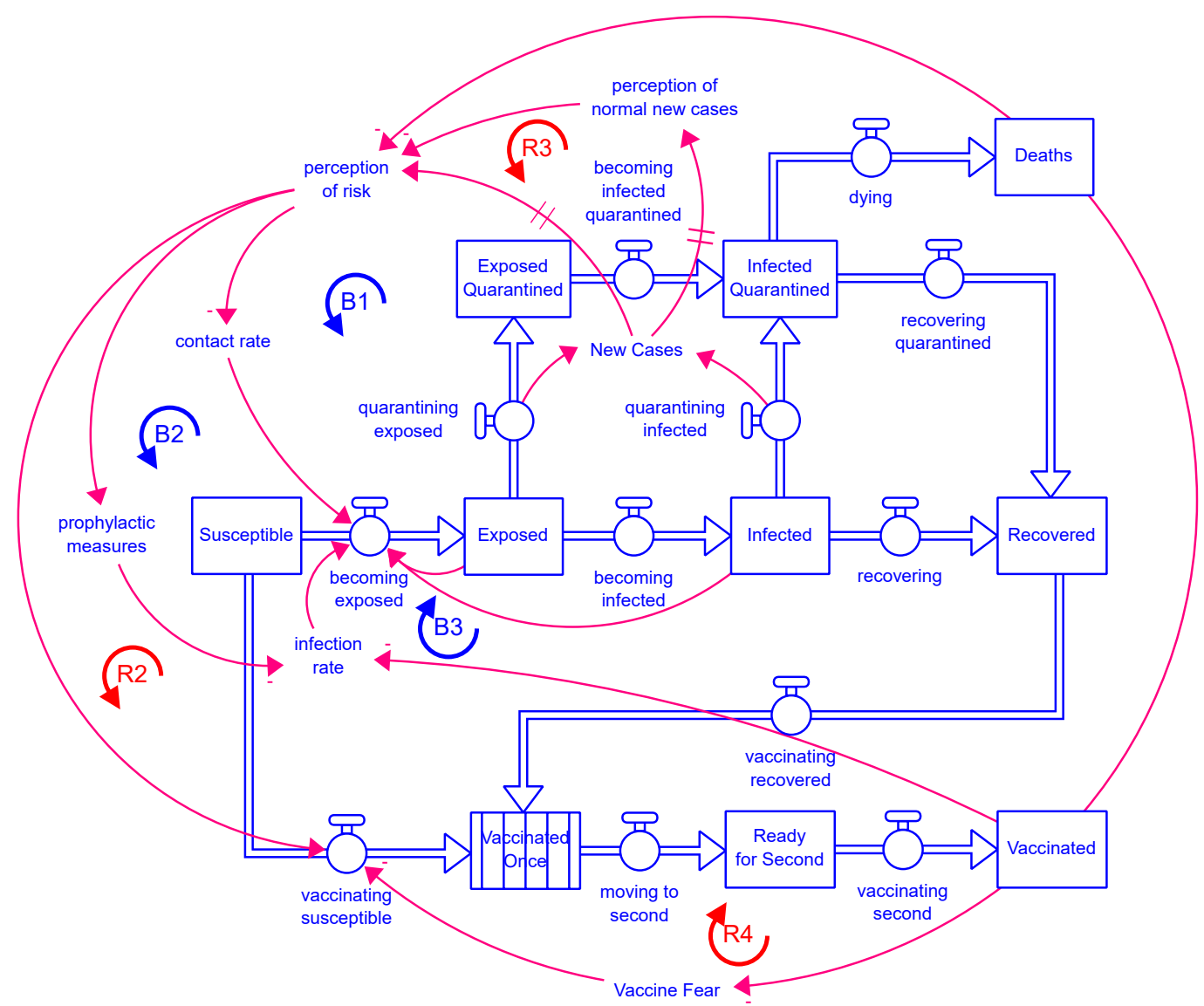

Figure 7. Full system diagram including all behavioral loops. All connections to vaccinating susceptible also go to vaccinating recovered.

Finally, the standard SEIR model uses first-order material delays-a single-stock delay in the movement of people through the system-to move between the stocks. This creates a distribution of people exiting such that the most people exit immediately, followed by a long tail. This distribution is not realistic, so a third-order material delay was used (third 
order creates the delay by moving through three stocks). This creates a distribution of people exiting each stage that resembles a skewed right normal distribution, i.e., more people reside in the respective stock for a shorter time on average than the specified time (Figure 8).

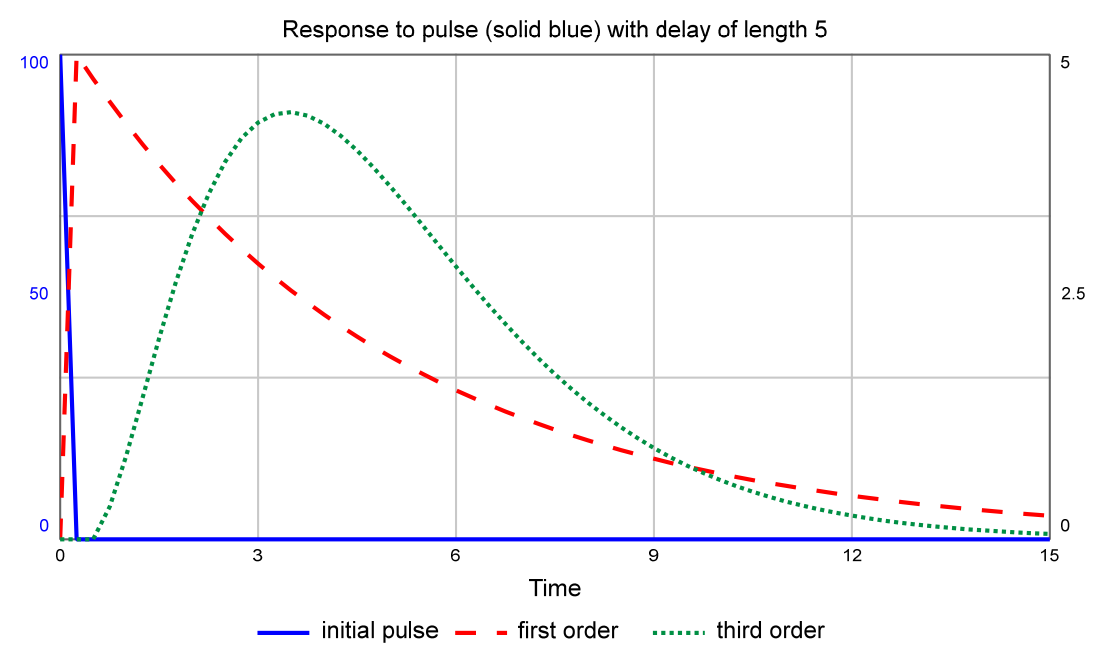

Figure 8. Pulse (solid blue, left axis) response of a first-order material delay (dashed red, right axis) and a third-order material delay (dotted green, right axis). Note the most material exits from the first-order delay in the first instant, i.e., there is only a DT delay.

\subsection{Model Behavior}

This model was calibrated for the USA data collected by the New York Times [11] between 13 January 2020 and 22 April 2021. The calibration was split across three regimes: 13 January to 6 June 2020, 6 June to 14 September 2020, and 14 September 2020 to 22 April 2021. These dates roughly correspond to the three waves of infection seen over the study period, as well as to changes in data accuracy, treatment protocols, and vaccine administration. Figure 9 shows the total cases for both the model (solid blue) and the data (dashed red), while Figure 10 shows the total number of deaths for both the model (solid blue) and the data (dashed red). It can be seen that, while not perfect, there is a fairly good match to the data $\left(R^{2}=0.999\right.$ for total cases and $R^{2}=0.998$ for deaths).

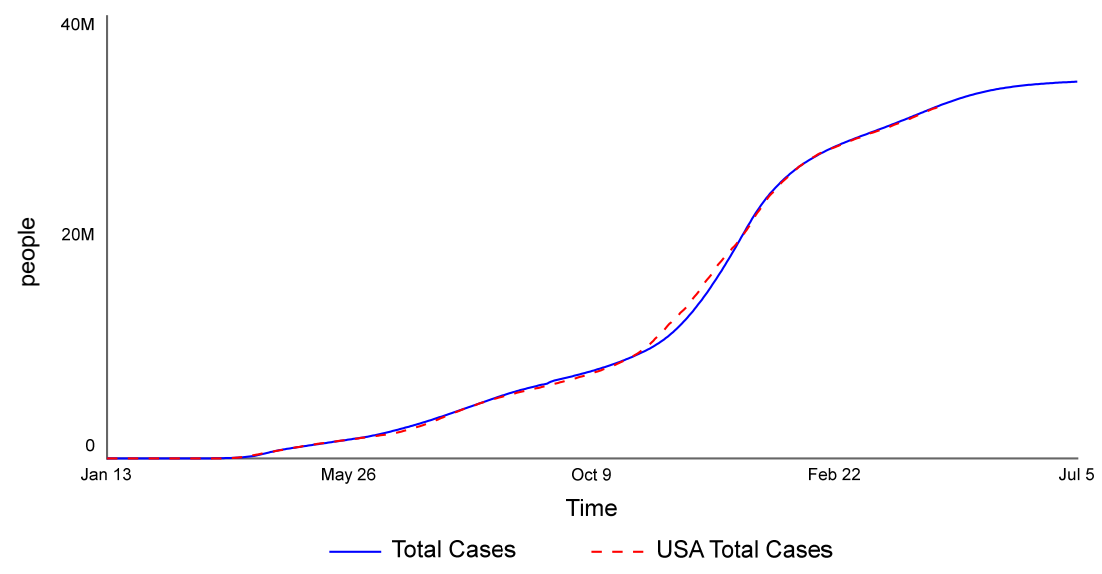

Figure 9. Model results (solid blue) and actual data (dashed red) for total cases in the USA. 


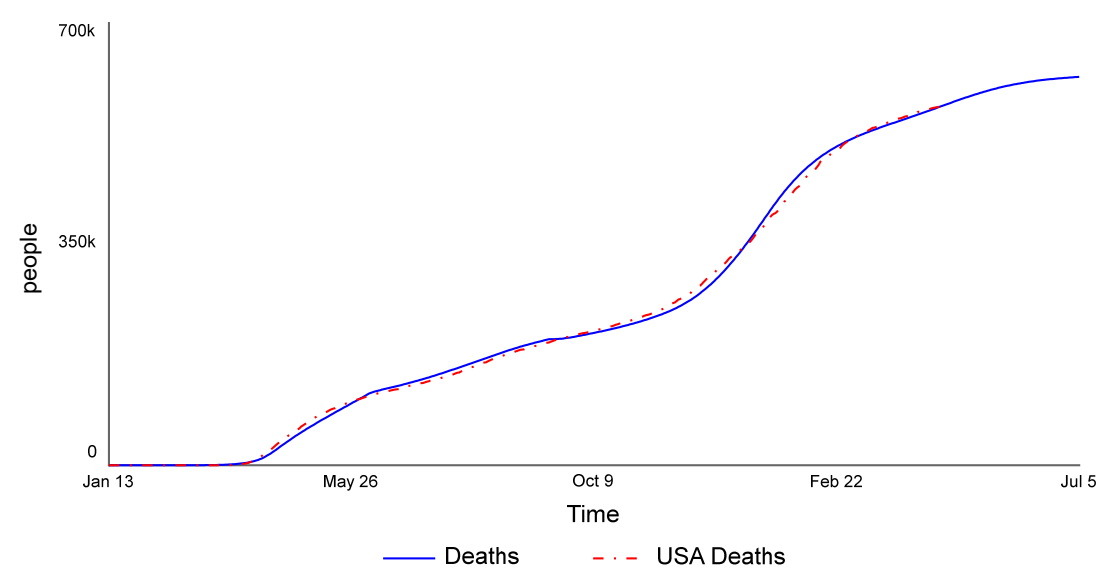

Figure 10. Model results (solid blue) and actual data (dashed red) for total deaths in the USA.

The full set of model equations appear in Appendix A. The dashboard to run the model is available here: https:/ / exchange.iseesystems.com/public/isee/covid-19-vaccinationsin-the-usa, accessed on 11 October 2021.

The impact of various policies was explored: (i) Removing the balancing behavioral loops from the model, i.e., B1, B2, and B3 in Figure 4; (ii) reducing prophylactic measures by some amount (10\% was arbitrarily chosen); (iii) removing masks starting 14 May 2021 per the CDC guidelines; (iv) delaying the arrival of the vaccine by an indefinite period; and (v) starting to vaccinate 12- to 15-year-old children on 16 May 2021. These policies were specifically chosen to exercise the behavioral aspects of the model.

\section{Results and Discussion}

\subsection{Impact of Behavioral Feedback Loops}

To quantify the impact of human behavior in this model, loops $B 1, B 2$, and $B 3$ in Figure 4 were disabled. Figure 11 shows the change in the number of cases against the base case that includes these loops. If people do not adapt their behavior to changing conditions and their changing perception of risk, i.e., if they always use masks and practice social distancing the same way regardless of how the pandemic progresses around them, the total cases over this period are much lower (roughly half). As a consequence, the deaths from COVID-19 (Figure 12) are also much lower (also roughly half).

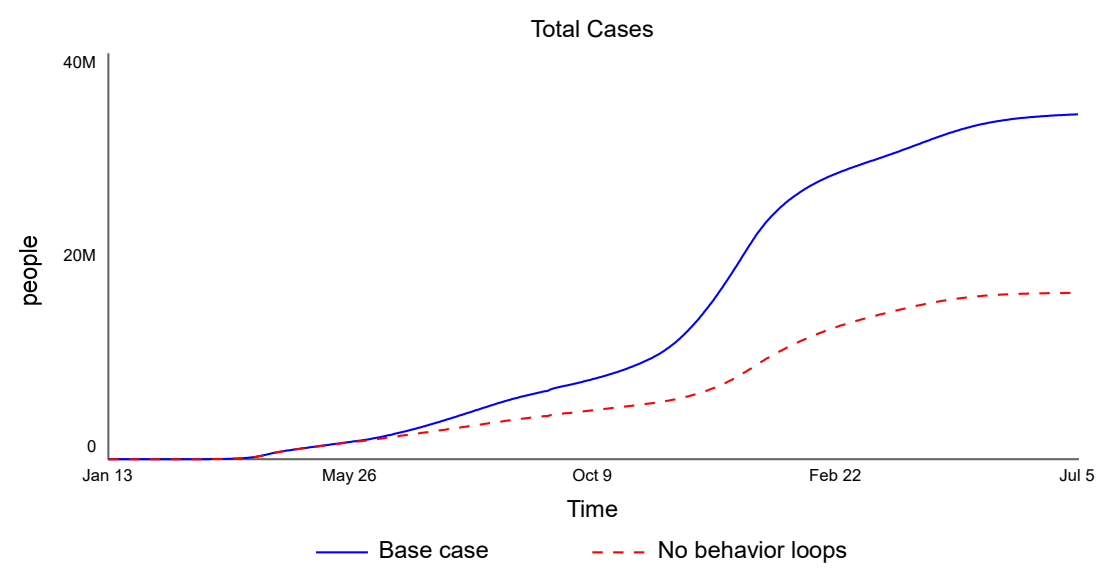

Figure 11. Change in the number of cases without behavioral balancing loops. 


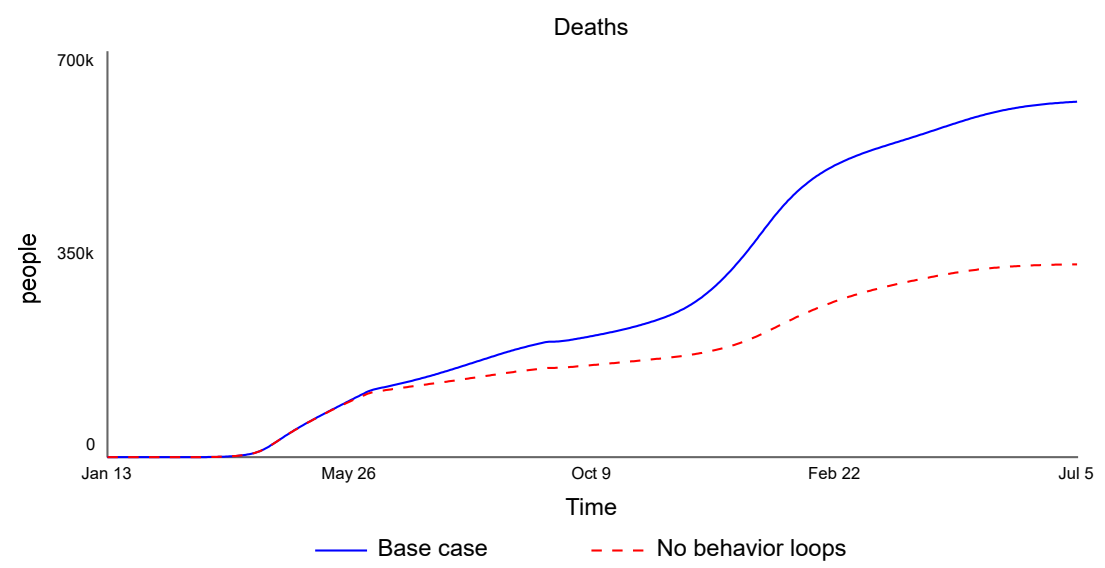

Figure 12. Change in the number of deaths without behavioral balancing loops.

Figures 11 and 12 both show a marked decrease in the rate of increase of cases and deaths during the holiday months (November through January) and then again after the vaccination program has taken off (February to July). While more people are in contact with each other during this time, the consistent use of masks and social distancing (without behavioral loops) reduces the spread of the disease. During the vaccination period, people remain vigilant as more people are vaccinated, maintaining their previous reduced levels of contact with others.

These results strongly suggest that human behavior is a critical aspect in controlling a pandemic. Outbreak models, such as the SEIR model, should include these aspects to provide better information to decisionmakers.

\subsection{Impact of People Being Less Careful}

Our second scenario tested the impact of people being slightly less careful with masks and social distancing, in particular, using them $10 \%$ less of the time than in the calibrated model. Based on what is known of the spread of COVID-19, one would expect the number of cases to somewhat increase. However, they increase quite substantially, almost $50 \%$ higher in January 2021 and approximately 25\% higher at the end of the simulation (Figure 13). The number of deaths follows a similar pattern.

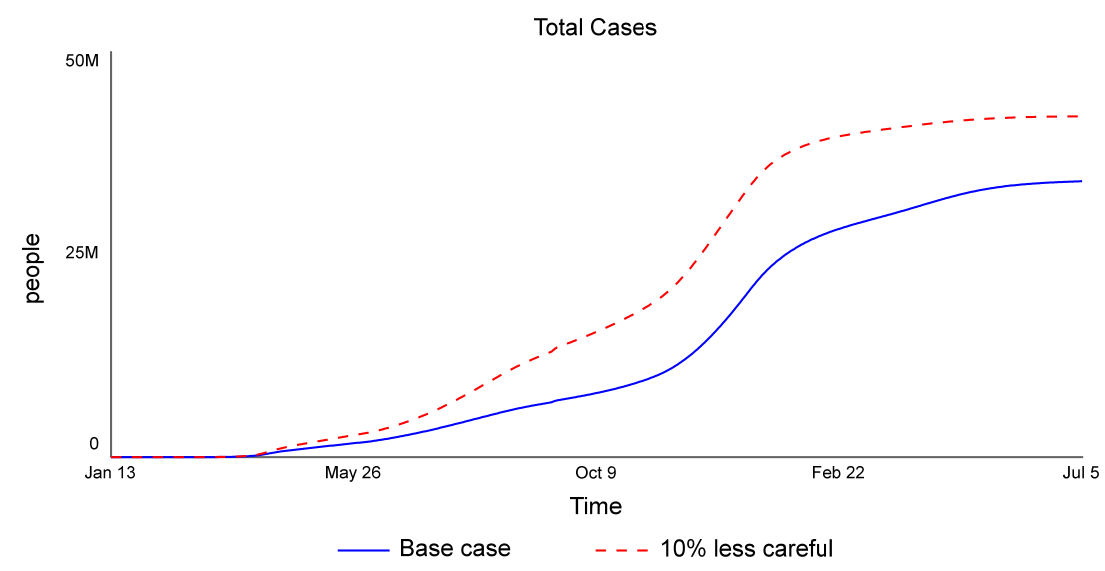

Figure 13. Change in total cases when the population is $10 \%$ less careful with masks and social distancing.

Beginning in June 2020, a self-reinforcing effect occurs involving feedback loop R3 in Figure 5, the drifting goals pattern. Because the number of new cases is larger than in the base case, the level of new cases considered dangerous also increases, further reducing people's diligence in not spreading the disease. This feedback loop continues to reinforce the already reduced diligence, reducing it further every time new cases increase in each new wave (summer 2020 and the 2020-2021 holiday season). Due to the faster spread of 
the disease, the maximum goal is also reached earlier, diluting this feedback loop's impact by April 2021 (a few months ahead of the base case).

These results not only emphasize the importance of prophylactic measures, but point directly to the strength of the reinforcing infection feedback loop in the SEIR model. Small increases in the infection rate or the contact rate can have large consequences. Likewise, small decreases can have a great impact on reducing the spread of the disease.

\subsection{Impact of the No-Mask Policy Partway through the Vaccination Program}

The no-mask-if-vaccinated policy adopted by the CDC on 14 May 2021 assumed people would be honest about whether or not they were vaccinated. In effect, it was a no-mask-for-anyone policy, instituted long before enough vaccinations were administered to reach herd immunity, the point at which the disease cannot continue to spread because there are not enough remaining susceptible people (whether immunity was achieved through infection and recovery or through vaccination). While this can be shown to have increased both total cases and total deaths, the more interesting segment to examine is breakthrough cases and deaths among those already vaccinated, i.e., the impact of the disease spreading through those already vaccinated. Note that breakthrough cases are unavoidable whenever a vaccine is not $100 \%$ effective. Breakthrough cases not only reduce confidence in the vaccines (not included here), but, more importantly, delay the return to normal that so many people desire-and presumably get vaccinated-to achieve. Figure 14 shows the increase in breakthrough infections with the no-mask policy (approximately $20 \%$ ). Figure 15 shows the increase in deaths from these infections (approximately $13 \%$ ).

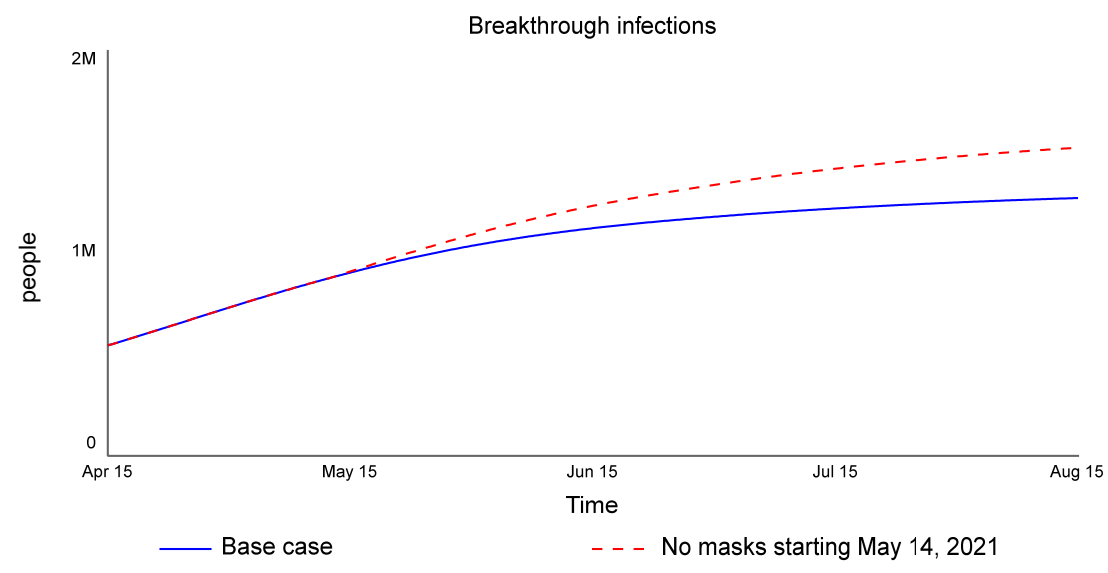

Figure 14. Change in breakthrough infections with a premature no-mask policy.

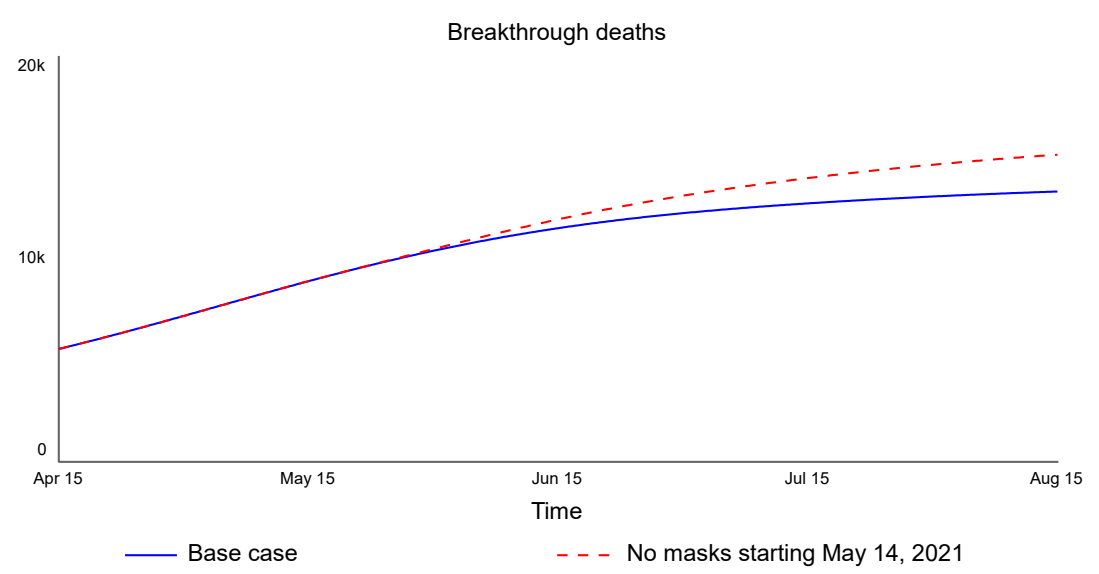

Figure 15. Change in deaths from breakthrough infections with a premature no-mask policy.

The combination of the vaccine not being $100 \%$ effective (in the model, it is $90 \%$ effective for all simulation runs) and higher unprotected contact between both unvaccinated 
and vaccinated people leads to an increase in breakthrough cases. Because the vaccine cannot fully protect against hospitalization and death, the number of deaths among those vaccinated also increases. The data for breakthrough infections is inadequate, so the absolute numbers given in these graphs are not accurate. However, they correctly reflect the behavior without masks, arguing for masks to be required once again among everyone (the CDC reversed the no-mask guidance on 28 July 2021).

\subsection{Impact of Delayed Vaccines}

Another scenario explored what would have happened if vaccinations in the USA could not start on 14 December 2020, but were delayed indefinitely. Figure 16 shows the increase in total cases by 5 July 2021 (approximately 9\%). The number of deaths follows a similar pattern.

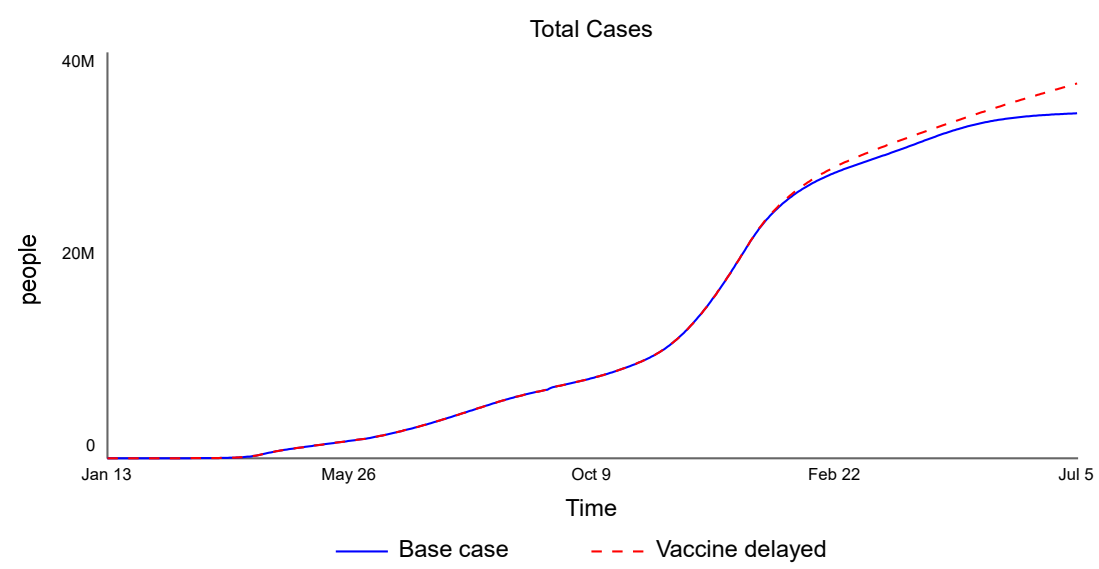

Figure 16. Change in total cases if the vaccine had been delayed.

Because the vaccine is not available, both feedback loop $R 2$ (people being less careful as more vaccines are administered) and $B 3$ (reduction in average infection rate due to vaccination) are inactive (see Figure 4 ). The lack of feedback $R 2$ indicates people will remain diligent longer, resulting in a lower contact rate than in the base case, while the lack of $B 3$ keeps the infection rate higher than in the base case. These somewhat balance each other out, so, in the short term, the spread of the infection does not get markedly worse without the vaccine. Note, however, that both the number of new cases and deaths trends upward much faster without the vaccine and will continue to grow at that accelerated rate until the vaccine is introduced.

The vaccination program was a necessary step to controlling the pandemic, but as the results from the scenarios above show, it is not enough. People need to get vaccinated and use both masks and social distancing until enough people are vaccinated to reach herd immunity.

\subsection{Impact of Approving the Vaccine for a New Age Group}

The final scenario tested the impact of vaccinating children aged 12-15 years starting 16 May 2021. Because of differences in both personal interactions and asymptomatic spread in different age groups, we would expect a reduction in the total number of cases and deaths. However, this model does not disaggregate by age, so those effects cannot be seen. Instead, the model increases the number of people eligible for the vaccine. As a result, what is apparent is the increase in the number of people vaccinated (Figure 17). 


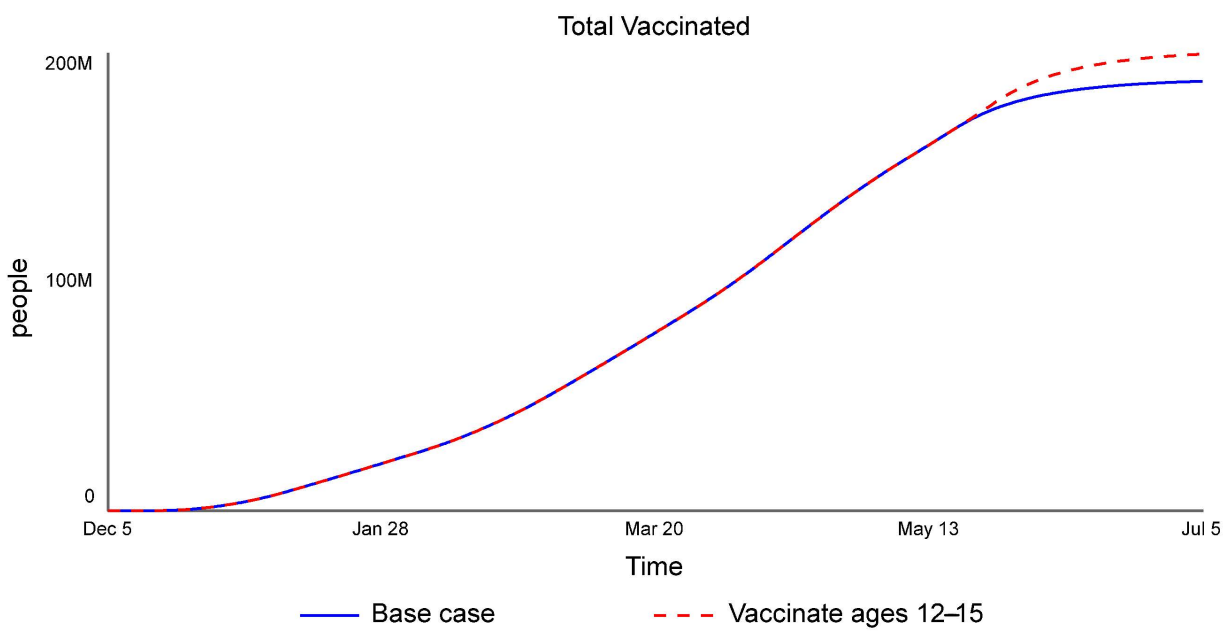

Figure 17. Increase in total vaccinations when including ages $12-15$ years.

While the model does not show the impact based on age, it does show that making more people eligible for the vaccine increases the number of people vaccinated. This, in turn, brings everyone closer to herd immunity.

\subsection{Summary}

Table 1 shows the values at the end of the simulation for the base case (no policies applied) and for each of the five policies studied. Note these are not the most important measures for policies 3 and 5 , which have a larger impact on breakthrough infections and total vaccinations, respectively.

Table 1. Value of total cases and deaths at the end of the simulation (5 July) for each of the five policies, as well as the percent change from the base case (no policy).

\begin{tabular}{ccccc}
\hline Policy & $\begin{array}{c}\text { Total Cases } \\
\text { (Millions) }\end{array}$ & $\begin{array}{c}\text { \% Change } \\
\text { Total Cases }\end{array}$ & $\begin{array}{c}\text { Deaths } \\
\text { (Thousands) }\end{array}$ & $\begin{array}{c}\text { \% Change } \\
\text { Deaths }\end{array}$ \\
\hline Base case & 34.1 & & 615 & \\
1. No behavior feedback & 16.4 & -51.9 & 333 & -45.9 \\
2. People less careful & 42.1 & 23.5 & 817 & 32.8 \\
3. Remove masks early & 34.5 & 1.17 & 620 & 0.813 \\
4. Delayed vaccine & 37.0 & -8.50 & 653 & 6.18 \\
5. Vaccinate youth & 34.0 & -0.293 & 614 & -0.163 \\
\hline
\end{tabular}

\section{Conclusions}

This study explored the impact of human behavior on the spread of the COVID-19 pandemic in the USA, as well as the progress of the USA's vaccination program. The SEIR model developed demonstrates that human behavior can have a significant impact on how fast the pandemic spreads and how many people become infected and die. Additionally, it shows that while vaccines can provide individuals a sense of security, that feeling is false because a reduction in prophylactic measures before herd immunity is reached leads to more infections and more deaths, including within the vaccinated population. Social distancing and masking must continue for the entire duration of the vaccine program. The danger of not having vaccines was demonstrated, as was the impact of vaccinating as many individuals as possible in moving toward herd immunity. While exact data are sparse for some of the situations explored (particularly breakthrough infections), the feedback structure of the system dictates the behavior pattern shown. It is hoped that other nations can learn from the experience of the USA and the scenarios explored here.

The model used for this investigation, while useful, has limitations that point to future research directions: (i) The question of the loss of vaccine immunity and possible boosters 
was not addressed; (ii) the model aggregated all age groups together, which impacted the spread and severity of the disease; (iii) the model aggregated the entire USA together, so while there were isolated quarantines throughout the country over the period modeled, in the model, there was only one quarantine activated for the entire country, at the start of the pandemic; (iv) mutations were used as a linear function based on infections, so infectivity never rose abruptly, as was seen with the delta variant; (v) mutations had no impact on vaccine effectiveness; and (vi) the impact of infections on hospital capacity was not included.

Funding: This research received no external funding.

Institutional Review Board Statement: Not applicable.

Informed Consent Statement: Not applicable.

Data Availability Statement: Publicly available datasets were analyzed in this study. This data can be found here: https:/ / github.com/nytimes/covid-19-data, accessed on 11 October 2021.

Conflicts of Interest: The author declares no conflict of interest.

\section{Appendix A. Model Equations without Calibration Settings}

Stocks:

Deaths $=\int($ dying $[$ not_yet $]+$ dying $[$ vaccinated $]) d t$

Exposed $=\int$ (becoming_exposed - becoming_infected - quarantining_exposed $) d t$

Exposed_Quarantined $=\int$ (quarantining_exposed - becoming_infected_quarantined $) d t$

Infected $=\int$ (becoming_infected - recovering - quarantining_infected $) d t$

Infected_Quarantined $=\int$ (quarantining_infected + becoming_infected_quarantined

- recovering_quarantined $-d y i n g) d t$

Prevalent_Strain $=\int($ mutating $) d t$

Ready_for_Second $=\int($ moving_to_second - vaccinating_second $) d t$

Recovered $=\int$ (recovering + recovering_quarantined - vaccinating_recovered - losing_immunity $) d t$

Susceptible $=\int($ becoming_susceptible - becoming_exposed - vaccinating_susceptible $) d t$

Total_Cases $=\int($ adding_cases $) d t$

Vaccinated $=\int$ (vaccinating_second - losing_vaccine_immunity $) d t$

Vaccinated_Once $=\int$ (vaccinating_recovered_in + vaccinating_susceptible_in

- moving_to_second $) d t$

transit time $($ Vaccinated_Once $)=$ time_to_second_dose

Vaccine_Fear $=\int($-diminishing_fear $) d t$

Flows:

adding_cases $=$ New_Cases

becoming_exposed $=$ infection_rate $\times$ susceptible_contacts_with_infected

becoming_infected $=$ newly_infected_people

becoming_infected_quarantined $=$ Exposed_Quarantined $/($ time_to_show_symptoms $/ 2)$

becoming_susceptible[not_yet $]=0$

becoming_susceptible[vaccinated] $=(1$ - vaccine_effectiveness $) \times$ vaccinating_susceptible[not_yet] diminishing_fear $=$ IF TIME $>=$ vaccine_start THEN Vaccine_Fear / time_to_reduce_fear ELSE 0 dying $=$ DELAYN2OUT(total_infected_quarantining, 1//death_rate, 3, 0,

desired_recovering_quarantined)

moving_to_second $=$ conveyor outflow(Vaccinated_Once)

mutating $=$ mutation_switch $\times$ new_mutations_possible/time_to_mutate

quarantining_exposed $=$ DELAYN2OUT2ND(becoming_exposed,

time_to_show_symptoms, distribution, 0, desired_testing)

quarantining_infected $=$ DELAYN2OUT2ND(becoming_infected, time_to_recover, distribution, 0 , desired_quarantining)

recovering $=$ DELAYN2OUT(becoming_infected, time_to_recover, distribution,

0 , desired_quarantining)

recovering_quarantined $=$ DELAYN2OUT2ND(total_infected_quarantining, $1 / /$ death_rate, 3, 
0, desired_recovering_quarantined)

vaccinating_recovered $=$ IF Vaccine_Status =Vaccine_Status.not_yet

THEN recovered_vaccines_to_administer ELSE 0

vaccinating_recovered_in $=$ vaccinating_recovered [not_yet $]$

vaccinating_second $=$ second_doses_to_administer

vaccinating_susceptible $=$ IF Vaccine_Status =Vaccine_Status.not_yet

THEN susceptible_vaccines_to_administer ELSE 0

vaccinating_susceptible_in $=$ vaccine_effectiveness $\times$ vaccinating_susceptible $[$ not_yet]

Parameters and other variables:

asymptomatic_fraction $=0.698841227245$

base_infection_rate $=0.024$

base_prophylactic_reduction_fraction $=0.53$

base_quarantine_effectiveness $=0.7$

behavior_switch $=1$

contact_rate $=$ vaccinated_effect_on_contact_rate $\times$ seasonal_effect_on_contact_rate

$\times$ quarantine_effect_on_contact_rate $\times$ normal_contact_rate

death_rate $=$ overall_death_rate $/$ time_to_recover_quarantined

desired_quarantining = symptomatic_quarantine_effectiveness

$\times(1$ - asymptomatic_fraction $) \times$ becoming_infected

desired_recovering_quarantined $=$ Infected_Quarantined $/$ time_to_recover_quarantined

desired_testing $=$ test_effectiveness $\times$ probability_exposed $\times$ testing_resources_noninfected

distribution $=3$

fear_effect_on_vaccinations $=f($ Vaccine_Fear $)$ :

$(0.0,1.0000),(10.0,0.802192027621),(20.0,0.66959737847),(30.0,0.580716527147)$,

$(40.0,0.521137910797),(50.0,0.481201169942),(60.0,0.454430771974)$,

(70.0, 0.436486037575), (80.0, 0.424457322387), (90.0, 0.416394233468),

$(100.0,0.410989383333)$

first_dose_demand $=((1-$ vaccine_hesitancy_switch $)+$ vaccine_hesitancy_switch

$\times$ fear_effect_on_vaccinations $) \times n e w \_c a s e s \_e f f e c t \_o n \_v a c c i n a t i o n s \times$ first_doses_needed

first_dose_resources $=$ vaccine_resources - second_doses_to_administer

first_doses_needed $=$ vaccine_demand $\times$ Vaccine_Ready

first_doses_to_administer $=$ vaccination_switch $\times \mathrm{MIN}($ first_dose_resources,

first_dose_demand/time_to_administer)

fraction_12-15 $=0.05212$

fraction_16+ $=0.7683$

fraction_population_severe $=$ severe_infected $/$ Live_Population

fraction_ready_vaccinated $=$ Total_Vaccinated $/$ Vaccine_Ready

fraction_susceptible $=$ Susceptible[not_yet $] / /($ Susceptible $[$ not_yet $]+$ Recovered $[$ not_yet $])$

fraction_vaccinated $=$ Total_Vaccinated $/ /$ Live_Population

holiday_effect_on_contact_rate $=($ holiday_multiplier -1$) \times$ holiday_season +1

holiday_multiplier $=1.3$

holiday_season $=f($ TIME MOD 365):

$(280.0,0.000),(284.0,0.0176),(288.0,0.0322),(292.0,0.0534),(296.0,0.132)$,

$(300.0,0.303),(304.0,0.513),(308.0,0.763),(312.0,0.921),(316.0,0.987),(320.0,1.000)$,

$(324.0,1.000),(328.0,1.000),(332.0,1.000),(336.0,1.000),(340.0,1.000),(344.0,1.000)$,

$(348.0,1.000),(352.0,0.9668),(356.0,0.8134),(360.0,0.399),(364.0,0.2166)$,

$(368.0,0.108),(372.0,0.050),(376.0,0.0208),(380.0,0.000)$

infection_rate $=$ prophylactic_effect_on_infection_rate

$\times$ mutation_effect_on_infection_rate $\times$ base_infection_rate

Live_Population $=$ Total_Population - Deaths

maximum_fraction_vaccinated $=0.75$

Maximum_New_Cases_So_Far=MAXIMUM(New_Cases)

mutation_effect_on_death_rate $=f($ Prevalent_Strain $)$

$(0.000,1.0000),(0.300,1.00976498428),(0.600,1.02428602211),(0.900,1.04458679859)$, 
$(1.200,1.07050171822),(1.500,1.1000),(1.800,1.12949828178),(2.100,1.15541320141)$,

$(2.400,1.17571397789),(2.700,1.19023501572),(3.000,1.2000)$

mutation_effect_on_infection_rate $=f($ Prevalent_Strain $)$

$(0.000,1.0000),(0.300,1.00976498428),(0.600,1.02428602211),(0.900,1.04458679859)$,

$(1.200,1.07050171822),(1.500,1.1000),(1.800,1.12949828178),(2.100,1.15541320141)$,

$(2.400,1.17571397789),(2.700,1.19023501572),(3.000,1.2000)$

mutation_switch $=1$

New_Cases = SUM(quarantining_exposed + quarantining_infected $)$

$n e w \_c a s e s \_e f f e c t \_o n \_p r o p h y l a c t i c s=f($ perception_of_new_cases $)$

$(0.000,0.000),(0.100,0.204704191101),(0.200,0.373647966026),(0.300,0.51307842386)$,

$(0.400,0.628151338387),(0.500,0.723121805124),(0.600,0.801501583626)$,

$(0.700,0.866188953175),(0.800,0.919575883568),(0.900,0.963636483909),(1.000,1.000)$

$n e w \_c a s e s \_e f f e c t \_o n \_q u a r a n t i n e=f($ perception_of_new_cases)

$(0.000,0.8000),(0.100,0.902726242763),(0.200,0.952728473174)$,

$(0.300,0.97706717163),(0.400,0.98891408801),(0.500,0.994680601285)$,

$(0.600,0.99748746463),(0.700,0.998853711695),(0.800,0.999518735537)$,

$(0.900,0.999842437392),(1.000,1.0000)$

$n e w \_c a s e s \_e f f e c t \_o n \_v a c c i n a t i o n s=f($ perception_of_new_cases)

$(0.000,0.2000),(0.100,0.468664729336),(0.200,0.648756083073),(0.300,0.7694749276)$,

$(0.400,0.850395189021),(0.500,0.904637662382),(0.600,0.940997479623)$,

$(0.700,0.965370193989),(0.800,0.981707713005),(0.900,0.992659079504)$,

$(1.000,1.0000)$

new_mutations_possible $=($ Total_Exposed + Total_Infected $) \times$ probability_of_mutation

newly_infected_people $=$ DELAYN2OUT(becoming_exposed, time_to_show_symptoms,

distribution, 0, desired_testing)

normal_contact_rate $=10.255130942$

normal_time_to_reduce_fear $=36.5991817151$

overall_death_rate $=0.0696453653012$

perception_of_new_cases $=\mathrm{SMTH} 3$ (relative_fraction_of_new_cases,

time_to_perceive_new_cases, 0$)$

probability_exposed $=\mathrm{SUM}($ Exposed $) / \mathrm{SUM}($ Susceptible + Exposed $)$

probability_of_contact_with_susceptible = Susceptible/(Total_Population

-Quarantined_or_Died)

probability_of_mutation $=1 / 1000000$

prophylactic_effect_on_infection_rate $=$ IF $($ TIME $>$ quarantine_start $/ 2)$

AND ((TIME <= 487) OR (NOT remove_masks_switch))

THEN 1 - prophylactic_switch $\times$ prophylactic_reduction_fraction ELSE 1

prophylactic_effectiveness $=$ IF quarantine_in_effect THEN 1

ELSE 0.85 - reduced_prophylactic_use_switch $\times 0.085$

prophylactic_reduction_fraction $=n e w \_c a s e s \_e f f e c t \_o n \_p r o p h y l a c t i c s$

$\times$ prophylactic_effectiveness $\times$ base_prophylactic_reduction_fraction

prophylactic_switch $=1$

quarantine_effect_on_contact_rate $=1-$ Quarantine_In_Effect $\times$ quarantine_effectiveness

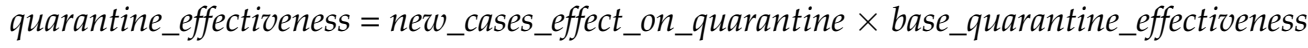

quarantine_in_effect $=($ TIME $>=$ quarantine_start $)$

AND (TIME < quarantine_start + quarantine_length)

quarantine_length $=64$

quarantine_start $=83$

Quarantined_or_Died $=$ Deaths + SUM $($ Exposed_Quarantined $)+\operatorname{SUM}($ Infected_Quarantined $)$

recovered_vaccines_to_administer $=$ first_doses_to_administer

- susceptible_vaccines_to_administer

reduced_prophylactic_use_switch $=0$

relative_fraction_of_new_cases = behavior_switch

$\times$ MIN(New_Cases//Maximum_New_Cases_So_Far, 1$)+(1$ - behavior_switch $)$ 
remove_masks_switch $=0$

seasonal_effect_on_contact_rate $=$ seasonality_switch $\times$ summer_effect_on_contact_rate

$\times$ holiday_effect_on_contact_rate $+(1-$ seasonality_switch $)$

seasonality_switch $=1$

second_doses_to_administer $=\mathrm{MIN}$ (vaccine_resources, Ready_for_Second $/$ time_to_administer)

severe_fraction $=0.2$

severe_infected $=$ severe_fraction $\times \operatorname{SUM}($ Infected_Quarantined $)$

summer_effect_on_contact_rate $=($ summer_multiplier -1$) \times$ summer_season +1

summer_multiplier $=1.1$

summer_season $=f($ TIME MOD 365)

$(150.00,0.000),(154.00,0.022),(158.00,0.088),(162.00,0.185),(166.00,0.386)$,

$(170.00,0.912),(174.00,1.000),(178.00,1.000),(182.00,1.000),(186.00,1.000)$,

$(190.00,1.000),(194.00,1.000),(198.00,1.000),(202.00,1.000),(206.00,1.000)$,

$(210.00,0.917),(214.00,0.399),(218.00,0.171),(222.00,0.066),(226.00,0.026)$,

$(230.00,0.000)$

susceptible_contacts_with_infected = probability_of_contact_with_susceptible

$\times$ total_infected_contacts

susceptible_vaccines_to_administer $=$ fraction_susceptible $\times$ first_doses_to_administer

symptomatic_quarantine_effectiveness $=0.75$

test_effectiveness $=0.8$

testing_resources $=$ RAMP $(7092.2$, testing_start, testing_start +282$)$

testing_resources_noninfected $=\mathrm{MAX}($ testing_resources $-\mathrm{SUM}($ quarantining_infected $), 0)$

testing_start $=45$

time_to_administer $=1$

time_to_mutate $=2000$

time_to_perceive_new_cases $=30$

time_to_reach_target $=120$

time_to_recover $=18.6059231858$

time_to_recover_quarantined $=$ time_to_recover $/ 2$

time_to_reduce_fear $=v a c c i n a t i o n \_e f f e c t \_o n \_f e a r \_r e d u c t i o n \times n o r m a l \_t i m e \_t o \_r e d u c e f f e a r$

time_to_second_dose $=25$

time_to_show_symptoms $=9.91451735291$

total_eligible_exposed $=$ "fraction_16+" $\times($ Total_Exposed + Total_Infected $)$

Total_Exposed $=\operatorname{SUM}($ Exposed $)+\operatorname{SUM}($ Exposed_Quarantined $)$

Total_Infected $=\mathrm{SUM}($ Infected $)+\mathrm{SUM}($ Infected_Quarantined $)$

total_infected_contacts $=$ contact_rate $\times \operatorname{SUM}($ Exposed + Infected $)$

total_infected_quarantining $=$ quarantining_infected + becoming_infected_quarantined

Total_Population $=330000000$

Total_Recovered $=$ SUM $($ Recovered $)$

Total_Susceptible $=$ SUM(Susceptible)

Total_Vaccinated $=$ Exposed [vaccinated $]+$ Exposed_Quarantined[vaccinated]

+ Infected[vaccinated] + Infected_Quarantined[vaccinated] + Ready_for_Second

+ Recovered[vaccinated] + Susceptible[vaccinated] + Vaccinated + Vaccinated_Once

Total_Vaccinated_Infected $=$ Exposed[vaccinated $]+$ Exposed_Quarantined[vaccinated]

+ Infected[vaccinated] + Infected_Quarantined[vaccinated] + Recovered[vaccinated] vaccinate_12_to_15_switch $=0$

vaccinated_effect_on_contact_rate $=f($ fraction_vaccinated $)$

$(0.0000,1.00334642546),(0.0400,1.00899310498),(0.0800,1.02371293659)$,

$(0.1200,1.05960146101),(0.1600,1.13447071069),(0.2000,1.2500)$,

$(0.2400,1.36552928932),(0.2800,1.44039853899),(0.3200,1.47628706341)$,

$(0.3600,1.49100689502),(0.4000,1.49665357454)$

vaccination_effect_on_fear_reduction $=f($ Vaccinated_Once $/$ Live_Population $)$

$(0.0000,2.500),(0.0500,2.00548006905),(0.1000,1.67399344618)$,

$(0.1500,1.45179131787),(0.2000,1.30284477699),(0.2500,1.20300292485)$, 
$(0.3000,1.13607692993),(0.3500,1.09121509394),(0.4000,1.06114330597)$,

$(0.4500,1.04098558367),(0.5000,1.02747345833)$

vaccination_switch $=1$

vaccine_demand $=f($ fraction_ready_vaccinated $)$

$(0.000,1.000),(0.100,0.917),(0.200,0.732),(0.300,0.553),(0.400,0.333),(0.500,0.197)$,

$(0.600,0.105),(0.700,0.053),(0.800,0.026),(0.900,0.013),(1.000,0.000)$

vaccine_dose_target $=3000000$

vaccine_effectiveness $=0.9$

Vaccine_Eligible $=$ "fraction_16+" $\times$ Live_Population + vaccinate_12_to_15_switch

$\times$ STEP("fraction_12-15", 489) $\times$ Live_Population

vaccine_hesitancy_switch $=1$

Vaccine_Ready $=$ maximum_fraction_vaccinated $\times$ (Vaccine_Eligible - total_eligible_exposed $)$

vaccine_resources $=\mathrm{RAMP}($ vaccine_dose_target $/$ time_to_reach_target, vaccine_start, vaccine_start + time_to_reach_target)

vaccine_start $=336$

Graphical functions:

fear_effect_on_vaccinations: As fear increases, vaccinations fall.

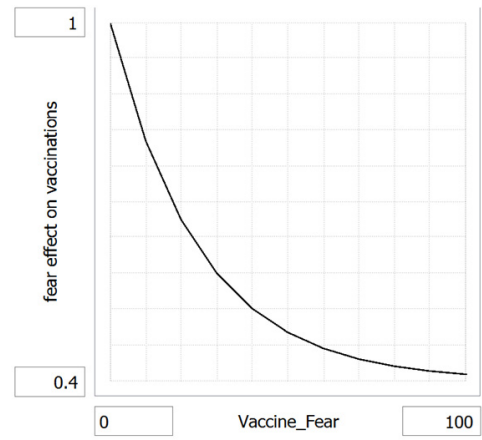

holiday_season:

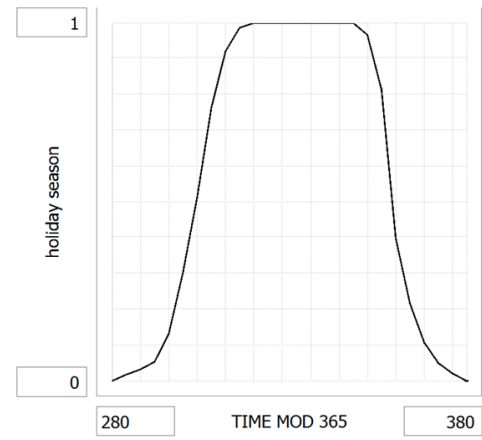

mutation_effect_on_death_rate: Later strains have higher death rates

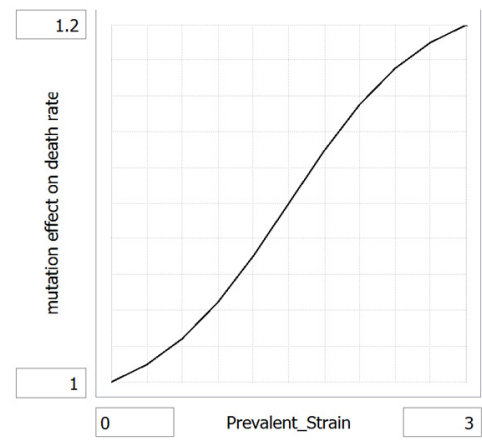


mutation_effect_on_infection_rate: Later strains have higher infection rates

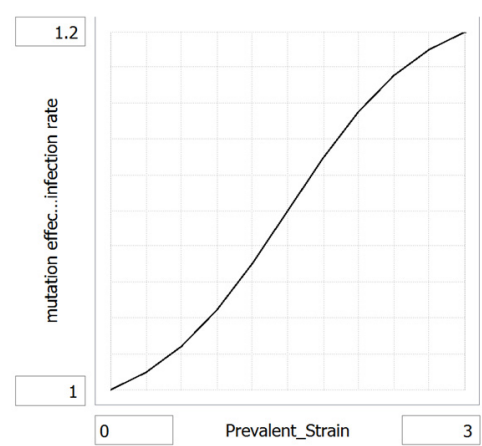

new_cases_effect_on_prophylactics: As perception of risk falls, use of prophylactics falls

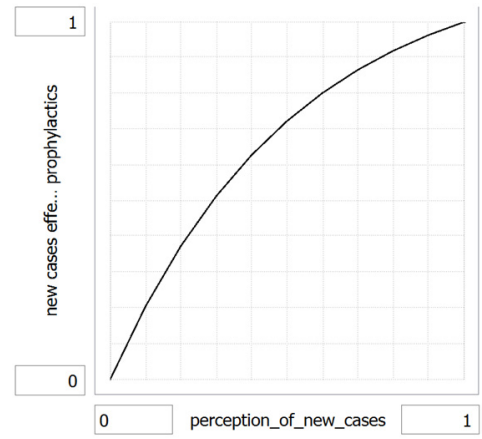

new_cases_effect_on_quarantine: As perception of risk falls beyond a certain point, the effectiveness of quarantine diminishes

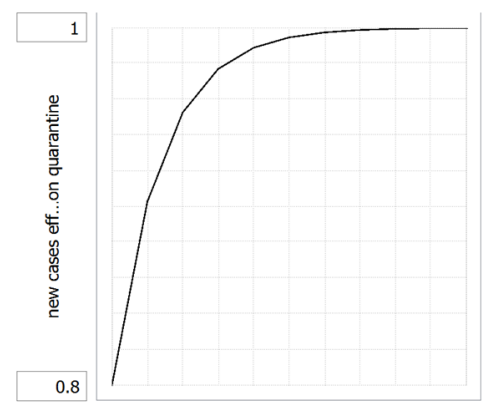

new_cases_effect_on_vaccinations: As perception of risk falls, the vaccination rate falls

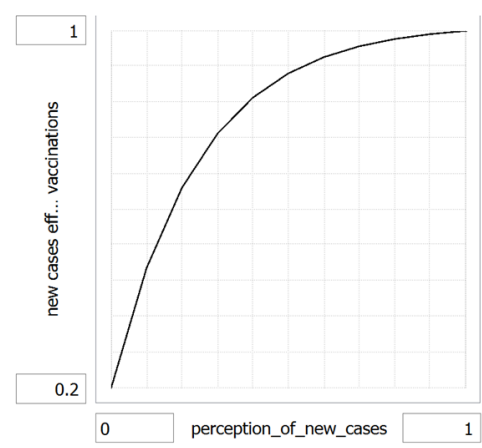


summer_season:

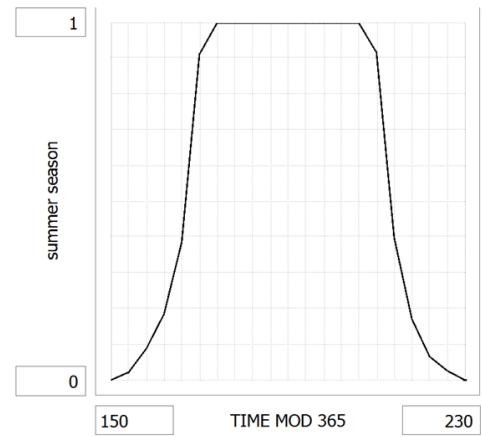

vaccinated_effect_on_contact_rate: As more people are vaccinated, the contact rate increases

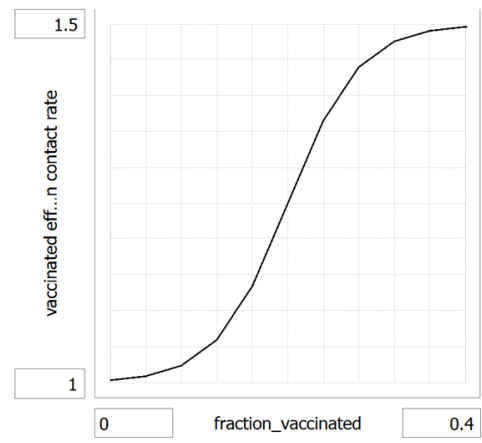

vaccination_effect_on_fear_reduction: As more people are vaccinated, the fear of the vaccine falls

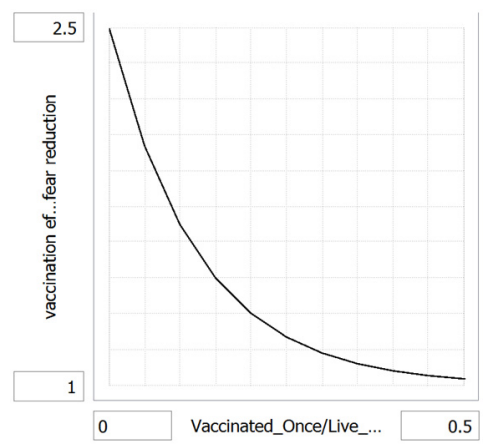

vaccine_demand: As more people are vaccinated, demand falls off

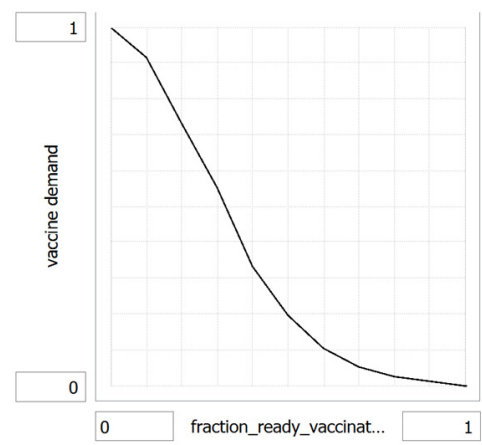




\section{References}

1. Struben, J. The coronavirus disease (COVID-19) pandemic: Simulation-based assessment of outbreak responses and postpeak strategies. Syst. Dynam. Rev. 2020, 36, 247-293. [CrossRef] [PubMed]

2. Ghaffarzadegan, N.; Rahmandad, H. Simulation-based estimation of the early spread of COVID-19 in Iran: Actual versus confirmed cases. Syst. Dynam. Rev. 2020, 36, 101-129. [CrossRef] [PubMed]

3. Sy, C.; Ching, P.M.; San Juan, J.L.; Bernardo, E.; Miguel, A.; Mayol, A.P.; Culaba, A.; Ubando, A.; Mutuc, J.E. Systems Dynamics Modeling of Pandemic Influenza for Strategic Policy Development: A Simulation-Based Analysis of the COVID-19 Case. Process. Integr. Optim. Sustain. 2021, 5, 461-474. [CrossRef]

4. Fair, J.M.; LeClaire, R.J.; Dauelsberg, L.R.; Ewers, M.; Pasqualini, D.; Cleland, T.; Rosenberger, W. Systems dynamics and the uncertainties of diagnostics, testing and contact tracing for COVID-19. Methods 2021, in press. [CrossRef] [PubMed]

5. Ferrar, R.; Klein, W.M. Risk perceptions and health behavior. Curr. Opin. Psychol. 2015, 5, 85-89. [CrossRef] [PubMed]

6. Bogdewic, S.; Ramaswamy, R. Developing Complexity-Informed COVID-19 Responses to Optimize Community Well-Being: A Systems Thinking Approach. Systems 2021, 9, 68. [CrossRef]

7. Rahmandad, H.; Lim, T.Y.; Sterman, J. Behavioral dynamics of COVID-19: Estimating under-reporting, multiple waves, and adherence fatigue across 92 nations. Syst. Dynam. Rev. 2021, 37, 5-31. [CrossRef] [PubMed]

8. Forrester, J.W. Industrial Dynamics; MIT Press: Cambridge, MA, USA, 1961.

9. Kermack, W.O.; McKendrick, A.G. A contribution to the mathematical theory of epidemics. Proc. R. Soc. Lond. A 1927, 115, 700-721. [CrossRef]

10. Senge, P.M. The Fifth Discipline: The Art and Practice of the Learning Organization; Doubleday/Currency: New York, NY, USA, 1990.

11. New York Times, COVID-19 Data. Available online: https:/ / github.com/nytimes/covid-19-data (accessed on 23 April 2021). 